\title{
MULTI-ROBOT PATH PLANNING BY PREDICTING STRUCTURE IN A DYNAMIC ENVIRONMENT
}

\author{
C. Spence Oliver ${ }^{1,2}$ Mahesh Saptharishi $^{1} \quad$ John M. Dolan ${ }^{2,3}$ \\ coliver@andrew.cmu.edu mahesh@cs.cmu.edu_jmd@cs.cmu.edu \\ $\begin{array}{lll}\text { (412) } 268-5214 & \text { (412) } 268-7109 & \text { (412) } 268-7988\end{array}$
}

\section{Ashitey Trebi-Ollennu ${ }^{2,4}$ \\ ashitey@jpl.nasa.gov}

(818) 354-8402

1. Dept. of Electrical and Computer Engineering

2. Institute for Complex Engineered Systems

3. The Robotics Institute

Carnegie Mellon University

5000 Forbes Ave.

Pittsburgh, PA 15213
Pradeep K. Khosla
pkk@cs.cmu.edu

(412) 268-5090

\author{
4. Jet Propulsion Laboratory \\ California Institute of Technology \\ 4800 Oak Grove Drive \\ Mail-Stop 82-105 \\ Pasadena, California 91109-8099
}

\begin{abstract}
Path planning for multiple mobile robots is complicated by the presence of a dynamic environment, in which obstacles and other robots are moving. Centralized approaches are too computationally intensive for real-time response. Decoupled approaches which perform individual preplanning, conflict resolution, and reactive obstacle avoidance for each robot, can be globally inefficient. We propose a novel mission coordination architecture, CPAD (Checkpoint/Priority/ Action Database), which performs path planning via checkpoint and dynamic priority assignment, using statistical estimates of the environment's motion structure in order to make both preplanning and reactive behaviors more efficient. Simulation is used to validate and illustrate the approach.
\end{abstract}

Keywords: multiple mobile robots, statistical path planning, mission planning, cooperation, path and structure estimation, predicting uncertainty, checkpoints, unsupervised clustering, neural nets, on-line learning

Invited paper for session: Collaboration and Data Fusion in Distributed Mobile Robotic Systems Classification: G1

\section{INTRODUCTION}

A group of mobile robots operating autonomously in an outdoor environment must be able to move from initial to goal positions efficiently, without collisions with obstacles and other robots. This multiple-robot path planning problem is complicated by the presence of a dynamic environment, in which obstacles and other robots are moving. Effective approaches must allow real-time response to changing conditions and should fully exploit sensory information about the environment.

CMU's CyberScout project involves the use of multiple mobile (CyberScouts) and stationary sentries for autonomous reconnaissance and exploration [1, 2,3]. The sentries traverse unknown or partially known terrains, so that pre-planned paths cannot account for all the dynamics in the environment. Reactive behaviors are used to account for this uncertainty, but they are at best locally optimal and can be inefficient from a global planning and execution perspective. However, given the nondeterminism of the environment and the computational inefficiency of repetitive global re-planning, reactive behaviors are unavoidable. How can the use of reactive behaviors be made more efficient? If the structure of the dynamic environment can be predicted, then points of uncertainty along the planned path can be identified and a global context can be applied in choosing a reaction to the uncertainty. This paper describes a novel approach to this problem which combines the use of checkpoints (i.e., areas where motion conflict is likely) and dynamic priorities with statistical estimation of the structure of the dynamic environment.

\section{APPROACH}

This section describes our approach to the described pathplanning problem in two parts. Section 2.1 describes the CPAD (Checkpoint/Priority/Action Database) mission coordination architecture, which performs path planning via checkpoint and dynamic priority assignment, and section 2.2 describes the estimation of motion structure in the environment. The combination of these two methods gives an efficient solution to path-planning in dynamic environments.

\subsection{Multi-robot path planning in dynamic environments}

Approaches to the multiple-robot path-planning problem are typically classed as centralized or decoupled. Centralized approaches regard the group of robots as a single entity such that all paths are planned simultaneously in a composite configuration space. Decoupled approaches plan for each robot individually, then seek to resolve any resulting conflicts. Neither approach explicitly includes the notion of a dynamic environment, so changes in the environment require replanning. Given the need for replanning, centralized approaches require too extensive inter-robot communications and computation to be feasible for real-time response.

There are various decoupled methods for dealing with conflicting robot paths $[4,5,6]$. [4] uses velocity tuning of the robots to ensure collision avoidance in a known environment, but this method assumes a static environment for which exact velocity trajectories can be pre-calculated. In [5] traffic rules are proposed for use by each robot, but these are effective only 


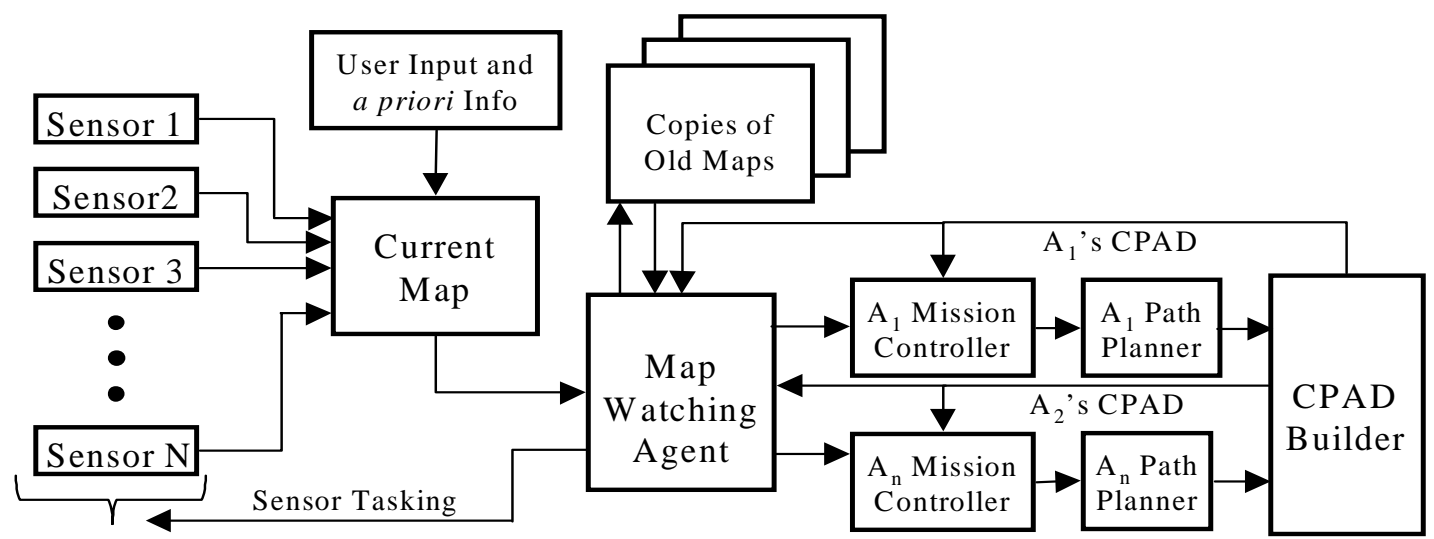

Figure 1. CPAD Mission Coordination Architecture for the CyberScout System

when adhered to by all moving objects in the environment. In [6] a framework is presented for merging multiple path plans in an incremental fashion. Using this methodology, however, a given robot may have to wait an unpredictable amount of time on a higher-prioritized robot before proceeding along its path, slowing down mission completion. The proposed CPAD mission architecture described in the following subsection avoids these problems.

\subsubsection{CPAD: Checkpoint/Priority/Action Database}

The goal of a CyberScout mission is to augment the human's ability to monitor a specified area. This is achieved by having the sensing agents distribute themselves throughout the workspace while gathering information about interesting activities. Missions may allow for autonomous or user-supervised operation, and must be structured to allow for a myriad of data collection and coordination commands in a constantly changing environment. We propose the Checkpoint/Priority/Action Database (CPAD) as a general framework for specifying such missions.

The CPAD is a collection of actions that must be performed by the CyberScouts to accomplish the surveillance mission. After the global surveillance task has been decomposed and distributed among the scouts, each scout plans its mission independently. An autonomous software agent analyzes the combination of plans and may recompute individual plans in such a way as to minimize conflict and maximize the probability of global mission success. From this global plan, the CPAD is constructed and distributed to the respective sensing agents. The CPAD is responsible for coordinating robot motions at possible intersections by assigning or updating relative priorities as new information is obtained. CPAD lends itself well to the coordination of multiple robots and previously detected moving object paths.

Figure 1 shows the architecture used to synthesize the distributed sensor data for predicting possible robot collisions. The CyberScout system begins with little or no a priori information about the workspace, $W$, while the human user selects an area or explicit points to be surveyed by the sensing agents. Task assignments are then centrally decomposed from the global specification and distributed to the individual robots,
$A_{1}, \ldots A_{\mathrm{n}}$, for planning. Each mobile scout $A_{\mathrm{i}}$ attempts to plan a path $\tau_{\mathrm{i}}$ through the workspace based on the limited knowledge available at the time. Because the workspace is represented by approximate cell decomposition, only approximate paths are planned for the scouts. The specifics of dealing with closequarter obstacle interactions are left to the local reactive planners used by each robot's mission controller. These local planners will attempt to steer a robot away from suddenly detected obstacles and back onto the global path. If a series of local reactions makes it impossible for the robot to reacquire its path, the mission controller instantiates its global path planner again.

Clearly, an individually planned path $\tau_{\mathrm{i}}$ may contain intersections with another such path $\tau_{j}$, where $j$ represents either a robot or a detected moving obstacle. Let $I_{(\mathrm{i}, \mathrm{j}, \mathrm{k})}$ denote the $\mathrm{k}^{\text {th }}$ possible intersection between two paths $\tau_{\mathrm{i}}$ and $\tau_{\mathrm{j}}$. The CPAD system coordinates multiple paths by first assigning a "soft" relative priority $p_{\mathrm{i}, \mathrm{j}}$ to each pair of robots $A_{\mathrm{i}, \mathrm{j}}$. At each possible intersection, checkpoints are placed around the intersecting region. If $A_{\mathrm{i}}$ has a higher priority than $A_{\mathrm{j}}$, then it has the "right-of-way" through the region and proceeds without stopping. If the lower-priority $A_{\mathrm{j}}$ approaches the checkpoint, it must stop and ensure that $A_{\mathrm{i}}$ is at a safe distance from the region before proceeding. Once $A_{\mathrm{j}}$ enters the intersecting region, the relative priorities between the two robots are reversed. Thus if $A_{\mathrm{j}}$ encounters unforeseen, temporary deadlock within the region, an approaching $A_{\mathrm{i}}$ will not constrain the space while $A_{\mathrm{j}}$ attempts to vacate the area. CPAD handles moving obstacle coordination in a similar way, except that an obstacle's "virtual path" (see section 2.2) is assigned the highest priority and can not be shifted lower.

The above conflict resolution scheme has a "first-come, firstserve" flavor to it, which raises the question, "why assign priorities at all?" Remember that CPAD is not just a path coordination technique, it is a general framework for specifying all aspects of a CyberScout's mission. Prioritized mission specification is part of the general framework of CPAD, and its use is exploited here. For a brief example of a necessary prioritization scheme, consider the overlapping of two paths $\tau_{1}$ and 


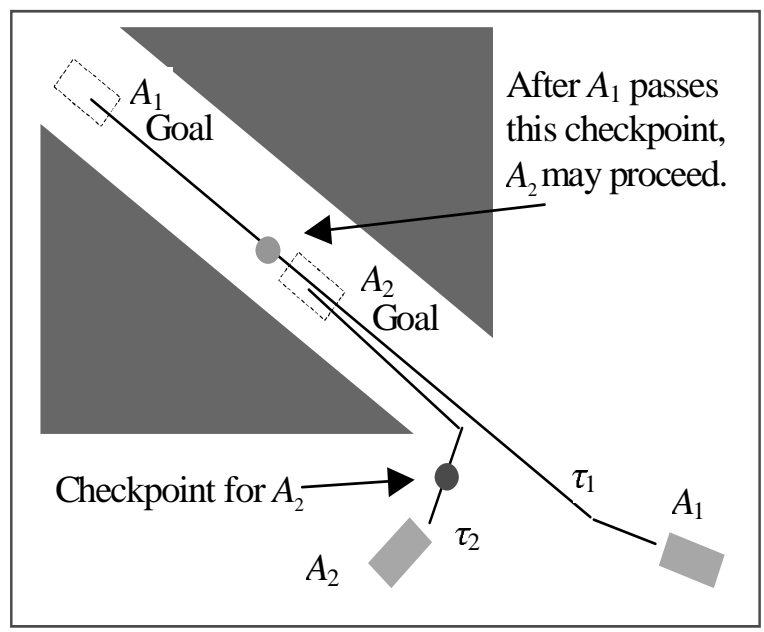

Figure 2. Path Coordination using CPAD

$\tau_{2}$, as shown in Figure 2. In order for both scouts to pass through the narrow corridor and achieve the proper goal allocation, $A_{2}$ must wait at a "hard-prioritized" checkpoint until $A_{1}$ is safely inside the corridor. Such a scenario is also useful for specifying automated convoying capabilities [1] which allow a scout with faulty or absent localization sensors to exploit the GPS (localization) information of a lead vehicle.

It is the responsibility of the CPAD builder in Figure 1 to analyze the collection of plans $\mathrm{T}=\left\{\tau_{\mathrm{i}}, \ldots, \tau_{\mathrm{n}}\right\}$, identify all possible intersections $I_{\mathrm{i}}=\left\{I_{(\mathrm{i}, 1,1)}, \ldots, I_{(\mathrm{i}, \mathrm{n}, \mathrm{k})}\right\}$ along a path $\tau_{\mathrm{i}}$, insert checkpoints appropriately around each intersection and assign relative priorites $p\left[A_{\mathrm{i}}, A_{\mathrm{j}}\right]$. Individual CPADs (or mission plans) are provided to the respective scouts, and the conglomerate CPAD is available to the autonomous map-watching agent described in section 2.2. The CPAD builder itself can be resident on any CyberScout or central command station through our distributed software infrastructure, CyberAries [3]. The map-watching agent identifies possible moving-obstacle trajectories that may intersect a path $\tau_{\mathrm{i}}$ and reports them to $A_{\mathrm{i}}$ 's mission controller. The mission controller then either adds the proper checkpoints to improve reactive behaviors near the obstacle or decides that a new path must be planned.

\subsection{Identifying Motion structure: The map watching agent}

Although relatively little work [7, 8, 9, 10] has been done in this area, Kruse, Gutsche and Wahl [7, 8] have recently considered analytical characterization of environmental motion dynamics in mobile-robot path-planning. They suggest an effective statistical method for estimating motion patterns and collision probabilities. We share a clear conceptual similarity to their approach, but we differ considerably in method and operating characteristics. First, we do not have constant coverage of the full environment. The network of scouts and stationary sentries constantly observe the environment within their sensors' field of view. These observations facilitate geolocation of visible moving objects in the environment, but the entire environment of interest may not be observable. A scout may spend anywhere from a very short to a considerable amount of time observing a particular piece of the operating region before planning. Thus, statistical assessments about the structure of the environment have to be made with very limited data. Second, Kruse et al. [8] adhere to the principle that "the robot is adapted to its environment, the environment is minimally disturbed by the robot." The size, appearance and sounds of the CyberScout inherently "disturb" the environment. Not only does the robot adapt to the the environment, the environment also adapts to the robot. As an illustrative example, consider walking through a crowded field. Not only do we take steps to efficiently reach our destination, the crowd also accommodates our actions to the best of its ability.

The motion structure we want to estimate is the set of paths that moving obstacles take in the environment. In other words, we wish to "beat a virtual path" in the map constructed by the scout. The virtual path indicates regions where the scout may possibly encounter moving obstacles. Virtual paths can be taken into account both during the preplanning phase and while the plan is executing. While the former use of the virtual paths attempts to eliminate uncertainty in the scout's path, the latter attempts to apply a global context in formulating a reaction to local uncertainty. Virtual paths can also be used to determine locations for surveillance.

Each scout is faced with computational and real-time constraints in all its actions. It performs many computationally intensive tasks such as surveillance, mission planning, obstacle avoidance, mapping, etc., at the same time. Thus, any additional processing tasks must work on a limited computational budget. Computationally intensive agents also reduce the sampling frequency of the environment. Given these constraints, all CyberScout agents attempt to process information that has a low spatial and temporal resolution with maximum efficiency. With computational parsimony in mind, the task of estimating and predicting motion structure in the environment uses residual data from the mapping algorithm to make its assessments. The mapping algorithm uses a Bayesian network [11] to identify static obstacles and constructs an occupancy grid of the observable environment. It also identifies moving obstacles and disregards them from its computations. The structure recognition algorithm to be described in this paper uses the moving obstacle data that the mapping algorithm identifies and disregards.

Each identified moving obstacle does not contain enough discriminating information for accurate temporal correspondence. Thus, estimation of potential virtual paths in the map depends purely on a temporally ordered sequence of moving obstacles. Each moving obstacle is represented by its location and size. The suggested approach shows that even with relatively little information about the moving obstacles in the environment, sufficient detail can be inferred to improve the quality of the planned path. We break our approach into two logical problems in statistics: estimation and prediction. We first estimate a highly probable topography of virtual paths given the observed samples of moving obstacles. After each 
estimation step, we predict points of uncertainty along the scout's planned path. Virtual paths are learned using unsupervised clustering of the positions of the moving obstacles using a mixture of Cauchy kernel functions. This technique is similar to Parzen Windows density estimation. The prediction step uses a neural network with the capability to perform selfsupervised diagnosis of itself and adapt online.

\subsubsection{Estimating the virtual paths}

Consider a set $S$ where each member is a set of moving obstacles observed at a particular time instance $t$. The members of set $S, m_{t}(S)$ are ordered temporally. We denote the $i^{t h}$ member of $m_{t}(S)$ as $m_{t, i}(S)$. Members of the set $S$ traverse paths in the environment which we shall denote as $P$. We choose to represent the members of $P$ as follows:

- Each member $m_{i}(P)$, a subpath in the network of virtual paths, maximizes $P\left(m_{i}(P) \mid\left\{m_{t}(S), m_{(t+1)}(S)\right\}\right)$, i.e., given two consecutive sets of moving obstacles, the subpath $m_{i}(P)$ explains the motion.

- We store $P\left(m_{i}(P) \mid\left\{m_{t}(S), m_{(t+1)}(S)\right\}\right)$, which is represented as a mixture of Cauchy kernels..

$$
P\left(m_{i}(P) \mid\left\{m_{t}(S), m_{(t+1)}(S)\right\}\right)=\sum_{i=1}^{n} \omega_{i} K\left(\vec{\mu}_{i}, C_{i}\right)
$$

The variable $n$ here represents the number of kernel functions in the mixture. $\vec{\mu}_{i}$ and $C_{i}$ represent the center and the covariance matrix respectively of the $i^{t h}$ kernel function in the mixture. $\omega i$ is the mixing coefficient for the $i^{\text {th }}$ kernel function.

The Cauchy kernel takes the general form:

$$
K=\frac{1}{1+\|x\|^{d+1}}, d \geq 0
$$

We use it in the following specific form:

$$
K(\vec{\mu}, C)=\frac{1}{1+\left\|(x-\vec{\mu})^{T} C^{-1}(x-\vec{\mu})\right\|}
$$

- Following the maximum of each "hill" created on the grid by each member of $P$, gives an estimate of the motion structure in the environment.

The Cauchy kernel was chosen because it exhibits the desired property of localized receptive fields. It was chosen over the Gaussian kernel because its tails fall off polynomially as opposed to exponentially in the case of Gaussian kernels. The covariance matrix $C$ determines the direction and extent of the spread of the kernel. The eigenvalues and eigenvectors of $C$ represent the two principal directions (assuming a 2D representation of the world) and magnitudes of the spread. Figures 3 and 4 illustrate an example of a Cauchy kernel explaning a pair of consecutive positions.

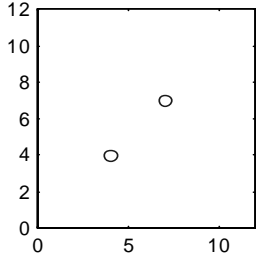

Figure 3



Figure 4
We now describe a greedy algorithm for choosing $n, \vec{\mu}_{i}, C_{i}$ and $\omega i$ so that $P\left(m(T) \mid\left\{m_{t}(S), m_{(t+1)}(S)\right\}\right)$ approximates the virtual paths given the observed data.

1. Initially the list of kernels is empty.

2. We consider the initial sets of moving obstacles $m_{0}(S)$ and $\mathrm{m}_{1}(\mathrm{~S})$.

Going forward in time:

3. Pick an obstacle $\mathrm{m}_{0,1}(\mathrm{~S})$ and pick $\mathrm{m}_{1, \mathrm{k}}(\mathrm{S})$ such that $\mathrm{m}_{1, \mathrm{k}}(\mathrm{S})$ is the closest obstacle to $\mathrm{m}_{0,1}(\mathrm{~S})$ in the set $\mathrm{m}_{1}(\mathrm{~S})$. Compute the mean and covariance matrix with respect to these points. Instantiate a kernel to explain this temporal pair. Record that this kernel has explained one temporal pair of obstacles.

4. Pick the next obstacle in $\mathrm{m}_{0}(\mathrm{~S})$. Pick the closest target to this obstacle in the set $\mathrm{m}_{1}(\mathrm{~S})$. Compute the mean and covariance matrix for this pair of data.

- Check if the obstacle under consideration falls within two standard deviations of any of the means of the kernels instantiated so far.

- If it does, then compute the eigenvalues and eigenvectors of the covariance matricies. Check if the eigenvalues of both matricies are ranked in the same order.

- If they are, check if the eigenvectors are pointing in similar directions.

- If all three of the previous conditions hold then we can explain the current data pair with a previously instantiated kernel. All we need to do is to recompute the mean and covariance matricies to better suit the data. Record that the kernel has explained another temporal pair. - If the conditions fail, then we instantiate a new kernel to explain the current temporal pair as in step 3 .

5. We repeat step 4 for all the obstacles in the set $m_{0}(S)$.

Going backwards in time:

6. We repeat step 4 for all the obstacles in the set $m_{1}(S)$. The assumption here is that if obstacles in $\mathrm{m}_{0}(\mathrm{~S})$ can move to $\mathrm{m}_{1}(\mathrm{~S})$, then the obstacles in $\mathrm{m}_{1}(\mathrm{~S})$ should be able to move to $\mathrm{m}_{0}(\mathrm{~S})$.

7. Weight each kernel based on the percentage of the total number of obstacles it successfully explains.

8. For all future sets $m_{t}(S)$ and $m_{t+1}(S)$, we repeat the above steps starting from step 4. 




Figure 5. Two obstacles moving on a road. The Scout's path intersects with the road.

This algorithm successfully hypothesizes a network of paths in the dynamic environment that best explains the data. We can regulate the complexity of the hypothesis in step 4 by specifying a smaller or larger number of standard deviations as the threshold for the proximity criteria. A smaller number of standard deviations leads to a more complex hypothesis while a larger number leads to a less complex hypothesis.

The following simulated example is representative of the results of the algorithm. There are two moving obstacles in the field of view. Figure 5 shows the positions of the obstacles through 4 time steps and the true path. The result of the algorithm after 4 time steps is shown in Figure 6 (3 kernels were used).

\subsubsection{Predicting uncertainty induced by virtual paths along planned paths}

After any estimation step, given a candidate path for the scout, we can determine if checkpoints are needed as a result of potential uncertainty. We use a single layer logistic linear network, trained with differential learning [12], to decide whether or not a checkpoint is needed. We then diagnose the neural network using discriminative diagnosis to determine the location in the path where a checkpoint is needed. Discriminative diagnosis was first described in the context of fault detection and diagnosis in [13]. It was extended as an online feature relevance estimation method and generalized to other applications in [14]. Discriminative diagnosis enables us to rank the relevance of the features listed in the feature vector given the output of the neural network to that feature vector. The input $X$ represents the virtual path $\mathrm{P}$ along the Scout's path. Given the input $\mathrm{X}$ and the decision of the neural network, discriminative diagnosis determines which cell in the vector $X$, contains the most uncertainty and thus requires a checkpoint. Simple thresholding of the input vector fails in the event of noise. Discriminative diagnosis also accounts for the temporal evolution of the virtual paths rather than considering each estimated path individually. Figure 6 shows the path of the Scout through the estimated virtual path and the chosen checkpoint. Figure 7

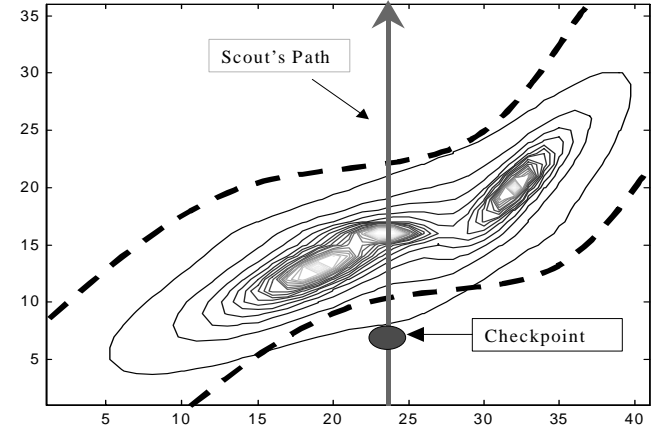

Figure 6. Contour plots show the set of virtual paths $\mathrm{P}$ estimated by the algorithm. The dashed lines indicate the true path. Given the values of the set $\mathrm{P}$ along the scout's path, the prediction algorithm suggests a checkpoint on the path.

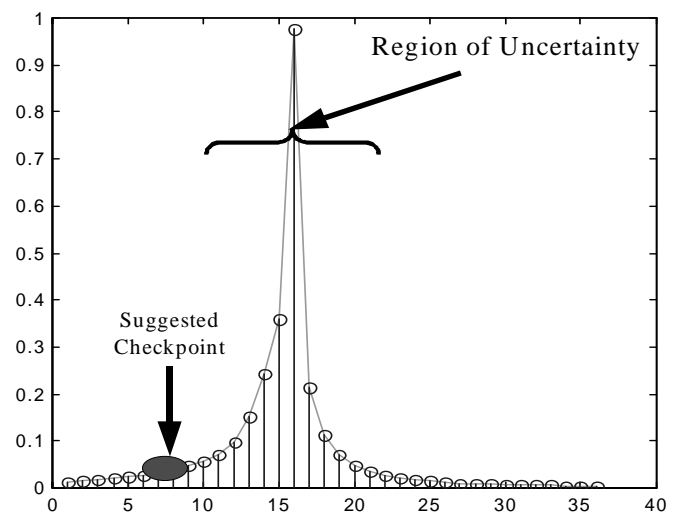

Figure 7. This plot shows the input to the network. The neural network identifies the region of uncertainty and suggests a checkpoint.

shows the input to the neural network, the detected region of uncertainty and the suggested checkpoint.

\section{RESULTS}

Our approach has been applied to various simulation data sets representing moving obstacles. Figures 8 and 9 present two scenarios for detecting virtual paths and coordinating the scouts' motion accordingly. In Figure 8, a stationary sentry detects a moving obstacle that will most likely intersect scout $A_{1}$ 's previously-planned path. The map-watching agent inserts a checkpoint at what it determines to be a safe distance from the virtual path. When $A_{1}$ arrives at the checkpoint, it will know to stop and dedicate additional perception efforts towards detecting the moving obstacle before proceeding.

Figure 9 presents the tasking of two scouts to locations suitable for observing an area. $A_{2}$ has the higher priority relative to $A_{1}$, but since $A_{1}$ enters the region surrounding the intersection $I_{(1,2,1)}$ first, it proceeds to its goal location. Once in position, $A_{1}$ is able to collect enough data to inform $A_{2}$ that a potential collision may occur along the path $\tau_{2}$. $A_{2}$ 's mission 


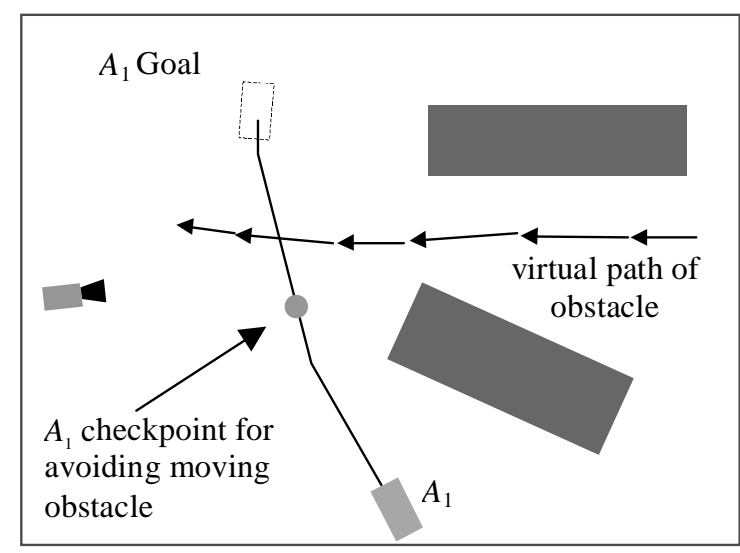

Figure 8. Insertion of Checkpoint by Virtual Path

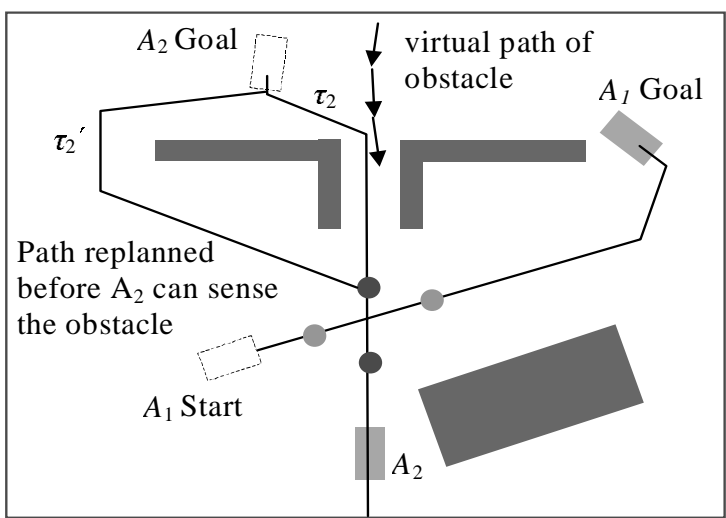

Figure 9. Reactive obstacle avoidance fails unless virtual path is reported to $A_{2}$

controller recognizes the need to replan and produces the new path $\tau_{2}$ '. Thus $A_{2}$ does not fall victim to local obstacle avoidance maneuvers in the corridor that will fail in the presence of the approaching obstacle.

\section{CONCLUSION}

We have proposed and demonstrated in simulation the effectiveness of a novel approach to path planning in dynamic environments using checkpoint and priority assignment in conjunction with statistical estimation of environmental motion structure. Future work will include the testing of this approach on real mobile robots in surveillance and other applications.

\section{REFERENCES}

[1] A. Soto, M. Saptharishi, J. Dolan, A. Trebi-Ollennu, and P. Khosla, "CyberATVs: Dynamic and Distributed Reconnaissance and Surveillance Using All-Terrain UGVs", Proceedings of the International Conference on Field and Service Robotics, pp. 329-334, Pittsburgh, PA, August 1999.

[2] J. Dolan, A. Trebi-Ollennu, A. Soto, and P. Khosla, "Distributed Tactical Surveillance with ATVs", SPIE Proceedings on Unmanned Ground Vehicle Technology, vol. 3693, pp. 192199, AeroSense ‘99, Orlando, FL, April 1999.
[3] C. Diehl, M. Saptharishi, J. Hampshire, and P. Khosla, "Collaborative Surveillance Using Both Fixed and Mobile Unattended Ground Sensor Platforms", SPIE Proceedings on Unattended Ground Sensor Technologies and Applications, vol. 3713, pp. 178-185, April 1999.

[4] K. Kant and S.W. Zucker, "Toward Efficient Trajectory Planning: Path Velocity Decomposition", International Journal of Robotics Research, vol. 5, pp. 72-89, 1986.

[5] S. Kato, S. Nishiyama, J. Takeno, "Coordinating mobile robots by applying traffic rules", IEEE International Conference on Intelligent Robots and Systems, vol. 3, pp 1535-????, IROS'92, Raleigh, (USA), July 1992.

[6] R. Alami, "A Multi-Robot Cooperation Scheme Based on Incremental Plan-Merging", Seventh International Symposium on Robotics Research, Giralt \& Gerhard (Eds.), Springer, 1995.

[7] E. Kruse and F.M. Wahl, "Camera-based Observation of Obstacle Motions to Derive Statistical Data for Mobile Robot Motion Planning", IEEE International Conference on Robotics and Automation, pp. 662-667, Leuven, Belgium, May 1998.

[8] E. Kruse, R. Gutsche, and F.M. Wahl, “Acquisition of Statistical Motion Patterns in Dynamic Environments and their Application to Mobile Robot Motion Planning", IEEE/RSJ International Conference on Intelligent Robots and Systems, pp. 712-717, 1997.

[9] E. Kruse, R. Gutsche, and F.M. Wahl, "Estimation of collision probabilities in dynamic environments for path planning with minimum collision probability", IEEE/RSJ International Conference on Intelligent Robots and Systems, pp. 1288-1295, 1996.

[10] R. Gutsche,C. Laloni and F.M.Wahl,'Path Planning for Mobile Vehicles Within Dynamic Worlds Using Statistical Data”, IEEE/RSJ International Conference on Intelligent Robots and Systems, pp. 454-461, 1994.

[11] E. Besada, J.A. Lopez-Orozco,'Data Fusion for Building Maps", Universidad Complutense de Madrid, CC. Fisicas. Tech Report Dacya 99/12.

[12] J.B. Hampshire II, B.V.K. Vijaya Kumar, "Differential Theory of Learning for Efficient Neural Network Pattern Recognition", SPIE Proceedings on the Science of Artificial Neural Networks, vol. 1966, pp 76-95, 1993.

[13] J.B. Hampshire II, D.A. Watola, "Diagnosing and Correcting System Anomalies with a Robust Classifier", International Conference on Acoustics, Speech and Signal Processing, pp 3507-3509, 1996.

[14] M. Saptharishi, "Differential Discriminative Diagnosis to Facilitate Online Learning With Neural Networks", MS Thesis, Dept. of Elec. and Comp. Engr., Carnegie Mellon University. To be submitted in 1999. 